\title{
SURGERY ON A CURVE IN A SOLID TORUS
}

\author{
BY
}

\section{J. P. NEUZIL}

\begin{abstract}
We consider the following surgery question: If a regular neighborhood of a polyhedral knot in a solid torus is removed and then sewn back differently, what manifold results? We consider two classes of knots, torus knots and what we call doubly twisted knots. We obtain some related results on surgery on knots in $s^{3}$.
\end{abstract}

1. Introduction. In this paper we consider surgery on a simple closed curve in a solid torus. That is, we consider the following question: If a regular neighborhood of a knot in a solid torus is removed and then sewn back differently, what manifold results? In particular, when is the manifold a solid torus?

A similar kind of surgery, surgery on knots in the 3-sphere, has been extensively studied. For a discussion of the history and importance of this problem, and for other references, see [7], [2], or [8]. Here we consider surgery on two kinds of knots, torus knots and what we call "doubly twisted knots." We also obtain some related results concerning surgery on knots in the 3-sphere.

2. Definitions and other preliminaries. In this paper, all manifolds, embeddings, regular neighborhoods, etc., will be piecewise linear. A solid torus is a 3-manifold-with-boundary homeomorphic to the product $S^{1} \times D^{2}$ of a circle and a disk. A torus is a 2-manifold-without-boundary homeomorphic to the boundary of a solid torus. A homotopy 3-cell is a compact simply connected 3-manifold whose boundary is a 2-sphere. A fake cube is a homotopy 3-cell which is not a 3-cell. A homotopy solid torus is an orientable 3-manifold-withboundary homeomorphic to a homotopy 3-cell with a pair of disks on its boundary identified. Note that the boundary of a homotopy solid torus is a torus and that if the homotopy 3-cell is a real 3-cell then the homotopy solid torus is a real solid torus. If $T$ is a homotopy solid torus, a meridian of $T$ is a simple closed curve on $\mathrm{Bd} T$ that bounds a disk in $T$ but is not null-homologous in $\mathrm{Bd} T$. A longitude of $T$ is a simple closed curve on $\mathrm{Bd} T$ which is transverse to a meridian. A core of a solid torus $T$ is a simple closed curve $h\left(S^{1} \times\{a\}\right)$ where $h$ is a homeomorphism of $S^{1} \times D^{2}$ onto $T$ and $a \in \operatorname{Int} D^{2}$. 
Let $T$ be an unknotted solid torus in $S^{3}$. Let $(M, L)$ be a meridianlongitude pair for $T$ such that $L$ bounds a disk in $S^{3}$ - Int $T$. Suppose also $K$ is a polyhedral simple closed curve in Int $T, T^{\prime}$ is a regular neighborhood of $K$ in Int $T$ and $(c, g)$ is a meridian-longitude pair for $T^{\prime}$. If $r$ and $s$ are relatively prime nonzero integers, we shall let $T_{g}(K: r, s)$ denote the orientable 3-manifold-with-boundary obtained from $T$ by $(r, s)$-surgery on $T^{\prime}$. That is, $T_{g}(K: r, s)$ is obtained from $T$ by removing Int $T^{\prime}$ and then sewing a solid torus $T^{\prime \prime}$ to $T$ - Int $T^{\prime}$ by a homeomorphism of $\mathrm{Bd} T^{\prime \prime}$ onto $\mathrm{Bd} T^{\prime}$ which takes a meridian of $T^{\prime \prime}$ to a curve on Bd $T^{\prime}$ homologous to $r c+s g$. If $g$ is homologous in $T$-Int $T^{\prime}$ to a multiple of $L$ then we abbreviate $T_{g}(K: r, s)$ to $T(K: r, s)$. Note that, for a particular knot $K$ in Int $T$, the family of 3 manifolds

$$
\left\{T_{g}(K: r, s):(r, s)=1\right\}
$$

is the same no matter what longitude $g$ is used. For, if $g$ and $g^{\prime}$ are longitudes transverse to the meridian $c$, then $g \sim k c+g^{\prime}(\sim$ means homologous with integer coefficients) for some integer $k$. Hence, $r c+s g \sim(r+s k) c+s g^{\prime}$ so that $T_{g}(K: r, s)$ is topologically equivalent to $T_{g^{\prime}}(K: r+s k, s)$. We shall use $G(K: r, s)$ to denote the group $\pi_{1}(T(K: r, s))$. By van Kampen's theorem,

$$
G(K: r, s) \cong \pi_{1}(T-K) / c^{r} g^{s}
$$

where the right side denotes $\pi_{1}(T-K)$ modulo the smallest normal subgroup containing $c^{r} g^{s}$.

Suppose $K$ is a knot in $S^{3},(c, g)$ is a meridian-longitude pair for $K$ and $(r, s)$ is a pair of relatively prime integers. We shall let $M_{g}^{3}(K: r, s)$ denote the manifold obtained by removing a regular neighborhood of $K$ from $S^{3}$ and sewing in a solid torus whose meridian is sewn to a simple closed curve homologous to $r c+s g$. If $g \sim 0$ in $S^{3}-K$ then we write $M^{3}(K: r, s)$ instead of $M_{g}^{3}(K: r, s)$. We say $K$ has property $\mathrm{P}$ if and only if $\pi_{1}\left(M^{3}(K: r, s)\right)$ is trivial only in the case $(r, s)=( \pm 1,0)$. It is known that a nontrivial knot $K$ has property $\mathbf{P}$ if and only if no counterexample to the Poincaré conjecture can be obtained by surgery on $K$ and the complement of $K$ in $S^{3}$ is unique among knot complements. See [2] or [8].

In [2], Bing and Martin considered surgery on certain knots in solid tori and we include their result for completeness.

THEOREM 1. If $K$ is a knot in the solid torus $T$ such that $K$ is not a core of $T$ and $T$ has as meridional disk which intersects $K$ exactly once, then the surgery manifold $T(K: r, s)$ is not a homotopy solid torus except in the trivial case $(r, s)=( \pm 1,0)$. 
We conclude this section with a lemma that characterizes homotopy solid tori algebraically. Before stating our lemma, we note the following fact: If $m$ and $n$ are relatively prime, then the group $G=\left\{a, b: a b=b a, a^{m} b^{n}=1\right\}$ is infinite cyclic. To show this, let $\{c\}$ be the free group on one generator and define homomorphisms $f:\{c\} \rightarrow G$ and $g: G \rightarrow\{c\}$ by $g\left(a^{i} b^{j}\right)=c^{n i-m j}$ and $f(c)=a^{\beta} b^{-\alpha}$ where $\alpha m+\beta n=1$. Then $f g$ and $g f$ are both the identity mappings.

Lemma 1. Suppose $T$ is an orientable 3-manifold such that $\mathrm{Bd} T$ is a torus and $\pi_{1}(T)$ is infinite cyclic. Then $T$ is a homotopy solid torus.

Proof. By the loop theorem, there is a simple closed curve $K$ on $B d T$ such that $K$ is homotopically nontrivial in $\mathrm{Bd} T$ and such that $K$ bounds a disk $D$ whose interior lies in Int $T$. Let $N_{1}$ be a regular neighborhood (in $T$ ) of $D$ such that $N_{1} \cap \mathrm{Bd} T$ is an annulus. Let $C_{1}=\mathrm{Cl}\left(T-N_{1}\right)$. Then $\mathrm{Bd} C_{1}$ is a 2-sphere. Let $N_{2}$ be a regular neighborhood (in $C_{1}$ ) of $\mathrm{Bd} C_{1}$. Let $C=$ $\mathrm{Cl}\left(C_{1}-N_{2}\right)$. Then $\mathrm{Bd} C$ is a 2 -sphere and we wish to show $C$ is simply connected. Now $T=C \cup\left(N_{1} \cup N_{2}\right), C \cap\left(N_{1} \cup N_{2}\right)=\operatorname{Bd} C=\operatorname{Bd}\left(N_{1} \cup N_{2}\right)$, and $N_{1} \cup N_{2}$ is a regular neighborhood of $D \cup \mathrm{Bd} T$. Also, $\pi_{1}(\mathrm{Bd} T)=$ $\{a, b: a b=b a\}$; therefore,

$$
\pi_{1}(D \cup \mathrm{Bd} T)=\left\{a, b: a b=b a, a^{m} b^{n}=1\right\}
$$

where $K$ represents the element $a^{m} b^{n}$ of $\pi_{1}(\mathrm{Bd} T)$. Since $K$ is a simple closed curve, $m$ and $n$ are relatively prime; hence $\pi_{1}\left(N_{1} \cup N_{2}\right) \cong \pi_{1}(D \cup \mathrm{Bd} T)$ is infinite cyclic. But $\left(N_{1} \cup N_{2}\right) \cap C$ is simply connected; therefore $\pi_{1}(T) \cong$ $\pi_{1}\left(N_{1} \cup N_{2}\right) * \pi_{1}(C)$, that is, the free product. But $\pi_{1}(T)$ and $\pi_{1}\left(N_{1} \cup N_{2}\right)$ are both infinite cyclic; hence $\pi_{1}(C)$ must be trivial. Therefore, $C$ is a homotopy 3-cell and $T$ is a homotopy solid torus.

3. Surgery on a torus knot in a solid torus. In this section we begin consideration of surgery on curves in solid tori. Our first case is that of a nicely embedded nontrivial torus knot. Throughout the rest of this paper, $T$ will refer to the standard unknotted solid torus in $S^{3}$. In this section, $K(p, q)$ will refer to a $(p, q)$ torus knot nicely embedded in $T$. That is, we assume there is a polyhedral annulus $A$ in $T$ such that one boundary component of $A$ is $K(p, q)$ and the other boundary component is a simple closed curve $K_{1}$ on $\mathrm{Bd} T$ which is homologous to $p M+q L$, where $M$ is a meridian of $T$ and $L$ is a longitude of $T$ which is null-homologous in $S^{3}$ - Int $T$. We may assume that $q>0$. By nontriviality, we mean that $K$ is not a core of $T$, hence $q \neq 1$. In the next theorem we will use the following construction: Let $T^{\prime}$ be a regular neighborhood of $K$ in Int $T$. We will assume that $T^{\prime}$ is constructed carefully enough 
so that $A_{1}=A \cap\left(T-\right.$ Int $\left.T^{\prime}\right)$ is an annulus, one of whose boundary components is $K_{1}$ and whose other boundary component is $A_{1} \cap T^{\prime}=A_{1} \cap \mathrm{Bd} T^{\prime}=$ $A \cap \operatorname{Bd} T^{\prime}$. We shall also assume there is a regular neighborhood $R$ of $A_{1}$ in $T$ - Int $T^{\prime}$ such that $A_{1} \cap \mathrm{Bd} R=\mathrm{Bd} A_{1}, R \cap \mathrm{Bd} T=\mathrm{Bd} R \cap \mathrm{Bd} T$ is an annulus with $K_{1}$ as a centerline, and $R \cap T^{\prime}=\mathrm{Bd} R \cap \mathrm{Bd} T^{\prime}$ is an annulus with $A_{1} \cap T^{\prime}$ as a centerline. In the following theorem we will use the longitude $d$ of $T^{\prime}$ where $d$ is a boundary component of the annulus $R \cap T^{\prime}$. Note that $d \sim A_{1} \cap T^{\prime}$ on $\mathrm{Bd} T^{\prime}$ and $d \sim K_{1}$ in $T$-Int $T^{\prime}$. It should also be noted that $d$ is, in general, not homologous (in $T-\operatorname{Int} T^{\prime}$ ) to any multiple of $L$ so that $T_{d}(K: r, s) \neq T(K: r, s)$. We shall assume, unless stated otherwise, that the surgery is nontrivial, that is, $(r, s) \neq( \pm 1,0)$.

THEOREM 2. If $K$ is a $(p, q)$ torus knot, $q>1$, embedded in $T$ as described above, then $T_{d}(K: r, s)$ is a union of two solid tori $T_{1}$ and $T_{2}$ such that $T_{1} \cap T_{2}$ is an annulus $B$ on the boundary of each and $B$ circles $\mathrm{Bd} T_{1}$ $q$ times longitudinally and circles $\mathrm{Bd} T_{2} r$ times longitudinally.

Proof. First we recall that $T_{d}(K: r, s)=\left(T-\operatorname{Int} T^{\prime}\right) \cup T^{\prime \prime}$ where $T^{\prime \prime} \cap$ $\left(T-\right.$ Int $\left.T^{\prime}\right)=\operatorname{Bd} T^{\prime \prime} \cap \operatorname{Bd}\left(T-\operatorname{Int} T^{\prime}\right)=\operatorname{Bd} T^{\prime}$ and a meridian of $T^{\prime \prime}$ is sewn to a curve homologous to $r c+s d$. ( $c$ is a meridian of $T^{\prime}$.) Let $T_{1}=$ $\mathrm{Cl}\left[T-\left(R \cup T^{\prime}\right)\right]$ and $T_{2}=R \cup T^{\prime \prime} . T_{1}$ is a solid torus since $R \cup T^{\prime}$ is a regular neighborhood of the curve $K_{1}$ on $\mathrm{Bd} T$. Next we show $T_{2}$ is a solid torus. Let $B_{1}=R \cap T^{\prime \prime} . B_{1}$ is an annulus with $A_{1} \cap T^{\prime \prime}$ as a centerline. Now $B_{1}$ circles $\operatorname{Bd} R$ exactly once longitudinally so $R$ is a regular neighborhood of the simple closed curve $A_{1} \cap T^{\prime \prime}$. Therefore $T_{2}$ is $T^{\prime \prime}$ plus a regular neighborhood of a curve on Bd $T^{\prime \prime}$. Hence $T_{2}$ is homeomorphic to $T^{\prime \prime}$.

It remains to show that the intersection of $T_{1}$ and $T_{2}$ is correct. Let $B=T_{1} \cap T_{2}$. Now $B=\left[R \cup T^{\prime}\right] \cap \mathrm{Cl}\left[T-\left(R \cup T^{\prime}\right)\right]=\mathrm{Cl}\left[\mathrm{Bd}\left(R \cup T^{\prime}\right)-\operatorname{Bd} T\right]$ which is an annulus since $\mathrm{Bd} T \cap \operatorname{Bd}\left(R \cup T^{\prime}\right)=\operatorname{Bd} T \cap \mathrm{Bd} R$ is an annulus. Now $d$ is a centerline of $B$ and $I$ circles $T q$ times longitudinally. Therefore $d$ (and hence $B$ ) circles $T_{1} q$ times longitudinally, since $T_{1}$ is a strong deformation retract of $T$. Also, there is a homeomorphism of $T_{2}=T^{\prime \prime} \cup R$ onto $T^{\prime \prime}$ which is the identity on $\mathrm{Cl}\left[\mathrm{Bd} T^{\prime \prime}-R\right]$ and which takes $\mathrm{Cl}\left[\mathrm{Bd} R-T^{\prime \prime}\right]$ onto $R \cap T^{\prime \prime}$. Since $d$ is a centerline of $B$ and the homeomorphism is the identity on $d$, it suffices to find the number of times $d$ circles $T^{\prime \prime}$ longitudinally. To do this, it suffices to find the (algebraic) intersection number of $d$ and a meridian of $T^{\prime \prime}$. Since a meridian of $T^{\prime \prime}$ is homologous to $r c+s d$, that intersection number is $r$. Hence $d$ circles $T^{\prime \prime} r$ times longitudinally and so $B$ circles $T_{2} r$ times longitudinally. This finishes the proof of Theorem 2.

As previously mentioned, $d \sim K_{1} \sim p M+q L$ in $T-$ Int $T^{\prime}$. Also, 
$M \sim q c$. Therefore $(-p q) c+d \sim-q L$. Hence, if $g$ denotes a longitude of $T^{\prime}$ which is homologous in $T$-Int $T^{\prime}$ to a multiple of $L, g \sim(-p q) c+d$ or $d \sim p q c+g$. Therefore, $r c+s d \sim r c+s p q c+s g$. Hence $T_{d}(K: r, s)=$ $T(K: r+s p q, s)$ and $T\left(K: r^{\prime}, s^{\prime}\right)=T_{d}\left(K: r^{\prime}-s^{\prime} p q, s^{\prime}\right)$.

Corollary 1. If $K=K(p, q)$ then

(i) $\pi_{1}\left(T_{d}(K: r, s)\right)=\left\{w, e: w^{r}=e^{q}\right\}$ and

(ii) $G(K: r, s)=\left\{w, e: w^{r-s p q}=e^{q}\right\}$.

This corollary follows directly from Theorem 2 by an application of van Kampen's theorem.

CoROllary 2. $T_{d}(K: r, s)$ is a homotopy solid torus if and only if $r= \pm 1 . T(K: r, s)$ is a homotopy solid torus if and only if $r-s p q= \pm 1$.

PROof. This corollary follows from Lemma 1 and the fact that $\pi_{1}\left(T_{d}(K: r, s)\right)$ is infinite cyclic if $r= \pm 1$ and is nonabelian if $|r|>1$.

Corollary 3. If $T_{d}(K: r, s)$ or $T(K: r, s)$ is a homotopy solid torus then it is a real torus.

Proof. If $T_{d}(K: r, s)$ is a homotopy solid torus then $r= \pm 1$. Thus $T_{d}(K: r, s)=T_{1} \cup T_{2}$ where $T_{1} \cap T_{2}$ is an annulus which circles $\mathrm{Bd} T_{2}$ exactly once longitudinally. Hence $T_{2}$ is a regular neighborhood of $T_{1} \cap T_{2}$, so $T_{1} \cup T_{2}$ is homeomorphic to $T_{1}$.

CoRollary 4. If $p$ is congruent to $r(\bmod q)$ and $n$ is congruent to $q(\bmod r)$ where $m$ and $n$ are integers such that $m r+n s=1$, then $T_{d}(K: r, s)$ is homeomorphic to a cube with an $(r, q)$ torus knotted hole and $T(K: r, s)$ is homeomorphic to a cube with an $(r-s p q, q)$ knotted hole.

A cube with an $(r, q)$ torus knotted hole is $\mathrm{Cl}\left[S^{3}-N(r, q)\right]$ where $N(r, q)$ is a regular neighborhood of an $(r, q)$ torus knot. Since the knot lies on the boundary of an unknotted solid torus, the cube with a hole may be written as $T_{1}^{*} \cup T_{2}^{*}$ where $B^{*}=T_{1}^{*} \cap T_{2}^{*}=\mathrm{Bd} T_{1}^{*} \cap \mathrm{Bd} T_{2}^{*}$ is an annulus which circles Bd $T_{1}^{*} q$ times longitudinally and $r$ times meridionally and which circles $\operatorname{Bd} T_{2}^{*}$ $r$ times longitudinally and $q$ times meridionally.

Proof of Corollary 4. By Theorem 2, $T_{d}(K: r, s)=T_{1} \cup T_{2}$ where $T_{1} \cap T_{2}$ is the annulus $B$ with $d$ as a centerline and $B$ circles $B d T_{1} q$ times longitudinally and circles $\mathrm{Bd} T_{2} r$ times longitudinally. Since $T_{1}$ is a solid torus contained in $T$ and a centerline of $T_{1}$ is also a centerline of $T$, there is a meridian-longitude pair $\left(M_{1}, L_{1}\right)$ for $T_{1}$ such that $d \sim p M_{1}+q L_{1}$.

Next we wish to find a meridian-longitude pair for $T_{2}$. Let $c_{2}$ be a 
meridian of the solid torus $T^{\prime} \cup R$ which bounds a disk $b_{2}$ in $T^{\prime} \cup R$ such that $b_{2} \cap \mathrm{Bd} T^{\prime}$ is the meridian $c$ of $T^{\prime}$. Also, $d$ is a longitude of $T^{\prime} \cup R$. Let $M^{\prime \prime}$ be a simple closed curve on $\mathrm{Bd} T^{\prime \prime}$ homologous to $r c+s d$. By construction, $M^{\prime \prime}$ is a meridian of $T^{\prime \prime}$; hence a simple closed curve $M_{2}$ on $\mathrm{Bd} T_{2}$ which is homologous to $r c_{2}+s d$ is a meridian of $T_{2}$.

Now there are integers $m$ and $n$ such that $m r+n s=1$. We claim a simple closed curve $L_{2}$ on $\mathrm{Bd} T_{2}$ such that $L_{2} \sim-n c_{2}+m d$ is a longitude for $T_{2}$. First $d \sim n M_{2}+r L_{2}$ and $c_{2} \sim m M_{2}-s L_{2}$, so $M_{2}$ and $L_{2}$ generate $H_{1}\left(\mathrm{Bd} T_{2}\right)$. Hence it suffices to show that the algebraic intersection number of $M_{1}$ and $L_{1}$ is 1 . Let $\#\left(J_{1}, J_{2}\right)$ stand for the algebraic intersection number of two oriented curves $J_{1}$ and $J_{2}$ on a torus. Then, since $M_{2} \sim r c_{2}+s d$, $\#\left(d, M_{2}\right)=r$ and $\#\left(c_{2}, M_{2}\right)=-s$ (with appropriate orientations). Hence $\#\left(L_{2}, M_{2}\right)=\#\left(-n c+m d, M_{2}\right)=-n(-s)+m r=1$.

Now we construct a homeomorphism which takes $T_{d}(K: r, s)$ onto $T_{1}^{*} \cup$ $T_{2}^{*}$. By hypothesis, there is an integer $n_{1}$ such that $r=p+n_{1} q$. Let $L_{1}^{\prime}$ be a simple closed curve on $\mathrm{Bd} T_{1}$ such that $L_{1}^{\prime} \sim n_{1} M_{1}+L_{1}$. Then $L_{1}^{\prime}$ is a longitude of $T_{1}$ and $d \sim r M_{1}+q L_{1}^{\prime}$. Therefore, since $d$ is a centerline of $B$, there is a homeomorphism $f_{1}$ of $T_{1}$ onto $T_{1}^{*}$ such that $\left(f_{1}\left(M_{1}\right), f_{1}\left(L_{1}^{\prime}\right)\right)$ is a meridian-longitude pair for $T_{1}^{*}$ and $f_{1}(B)=B^{*}$. Similarly, there is an integer $n_{2}$ such that $n=q+n_{2} r$. Let $L_{2}^{\prime}=n_{2} M_{1}+L_{2}$. Then $d \sim q M_{1}+r L_{2}^{\prime}$. Therefore, there is a homeomorphism $f_{2}$ of $T_{2}$ onto $T_{2}^{*}$ such that $\left(f_{2}\left(M_{2}\right)\right.$, $\left.f_{2}\left(L_{2}^{\prime}\right)\right)$ is a meridian-longitude pair for $T_{2}^{*}$ and $f_{2}$ agrees with $f_{1}$ on $B$. This concludes the proof of Corollary 4. then

LEMmA 2(a). If $r-s p q=1$ (and hence $T(K(p, q): r, s)$ is a solid torus)

$$
G(K(p, q): r, s)=\left\{b, x: b x=x b, x^{r} b^{s q^{2}}=1\right\},
$$

where $b$ is the element of $\pi_{1}(T(K: r, s))$ generated by the longitude $L$ and $x$ is the element generated by the meridian $M$. In addition, a simple closed curve homologous to $r M+s q^{2} L$ is a meridian of $T(K: r, s)$. If $r-s p q=-1$ then the conclusion is the same with $s$ replaced by $-s$.

Proof. Our first task is to construct a presentation for $\pi_{1}(T-K(p, q))$ in terms of $b, x$ and $y$ where $y$ is the element of the fundamental group generated by a core of $T$. Since $K$ is nicely embedded in $T$, there is a solid torus $T^{*}$ in Int $T$ such that $K$ lies on $\mathrm{Bd} T^{*}$ and such that a core of $T^{*}$ is also a core of $T$.

Let $S=\mathrm{Cl}\left(T-T^{*}\right)$. Then $S$ is homeomorphic to the product of a torus and an interval. Let $T^{\prime}$ be the regular neighborhood of $K$ as described at the 
beginning of this section. We shall assume that $T^{\prime} \cap T^{*}, T^{\prime} \cap S$ and $T^{\prime} \cap \mathrm{Bd} T^{*}$ are regular neighborhoods of $K$ in $T^{*}, S$ and $\mathrm{Bd} T^{*}$ respectively. We will find generators and relations for $\pi_{1}(T-K) \cong \pi_{1}\left(\mathrm{Cl}\left(T-T^{\prime}\right)\right)$ from $\pi_{1}\left(\mathrm{Cl}\left(T^{*}-T^{\prime}\right)\right)$ and $\pi_{1}\left(\mathrm{Cl}\left(R-T^{\prime}\right)\right)$ using van Kampen's theorem.

Now $\mathrm{Cl}\left(T^{*}-T^{\prime}\right)$ is a solid torus. Let $y$ be the element of $\pi_{1}\left(\mathrm{Cl}\left(T^{*}-T^{\prime}\right)\right)$ generated by a core of $T^{*}$ which misses $T^{\prime}$. Then $\pi_{1}\left(\mathrm{Cl}\left(T^{*}-T^{\prime}\right)\right)$ is a free group with generator $y$. Also, $\pi_{1}\left(\mathrm{Cl}\left(R-T^{\prime}\right)\right) \cong\{x, b: b x=x b\}$. Now

$$
\mathrm{Cl}\left(R-T^{\prime}\right) \cap \mathrm{Cl}\left(T^{*}-T^{\prime}\right)=\mathrm{Cl}\left(\mathrm{Bd} T^{*}-T^{\prime}\right)
$$

is an annulus which circles $T^{*} p$ times meridionally and $q$ times longitudinally. Let $z$ be a generator of $\pi_{1}\left(\mathrm{Cl}\left(\mathrm{Bd} T^{*}-T^{\prime}\right)\right)$. In $\pi_{1}\left(\mathrm{Cl}\left(T^{*}-T^{\prime}\right)\right), z=y^{q}$ and in $\pi_{1}\left(\mathrm{Cl}\left(R-T^{\prime}\right)\right), z=b^{q} x^{p}$. Therefore, by van Kampen's theorem,

$$
\pi_{1}(T-K(p, q)) \cong \pi_{1}\left(\mathrm{Cl}\left(T-T^{\prime}\right)\right) \cong\left\{b, x, y: b x=x b, y^{q}=b^{q} x^{p}\right\} .
$$

Our next task is to find a meridian-longitude pair for $K(p, q)$ in terms of $x, y$ and $b$. Our method is to use the overcrossing-undercrossing presentation of $T-K(p, q)$ in essentially the same way as J. Hempel did in [6]. Instead of using $\pi_{1}(T-K(p, q))$ directly, we will use the link group $\pi_{1}\left(S^{3}-\left(M^{*} \cup K(p, q)\right)\right)$ where $M^{*}$ is a curve in $S^{3}-T$ which is parallel to $M$.

Now, since $p$ and $q$ are relatively prime, there are integers $\alpha$ and $\beta$ such that $\alpha p+\beta q=1$ and $\beta>0$. Then $\pi_{1}\left(S^{3}-\left(M^{*} \cup K(p, q)\right)\right)$ has generators $a_{1}, \cdots, a_{p+q}$ (see Figure 1) and the following relations:

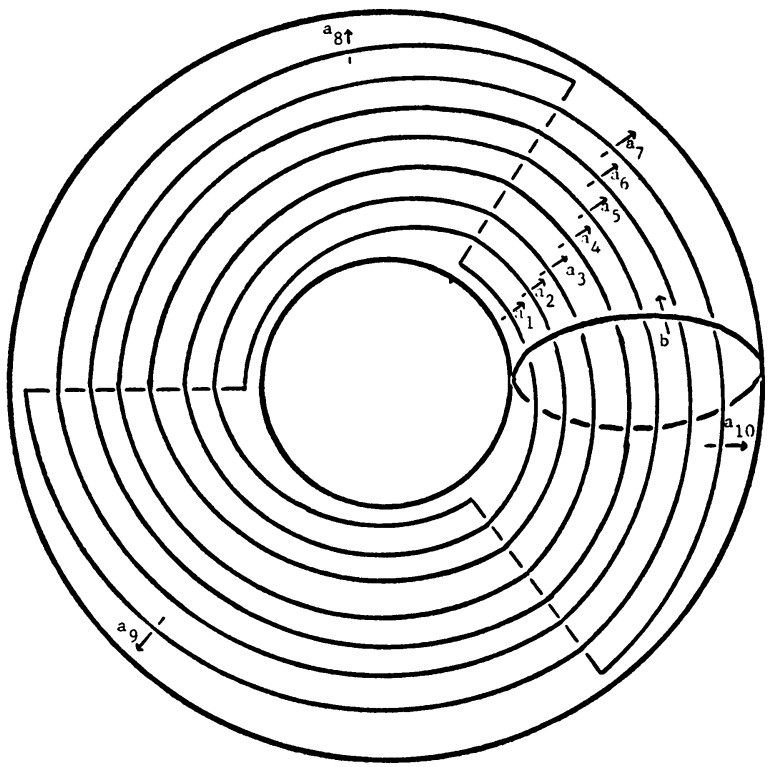

FiguRE 1 
(1) for $1 \leqslant k \leqslant p, a_{k} \cdots a_{k+q-1}=a_{k+1} \cdots a_{k+q}$, and

(2) if $k>p$ and $k=n p+j$ with $0 \leqslant j<p$ then $a_{k}=b^{n} a_{j} b^{-n}$ where we define $a_{0}=a_{p}$.

Since $y$ is represented by a core of $T^{*}, y=a_{1} \cdots a_{p} b$ and, since $x$ is represented by a curve parallel to $M, x=a_{1} \cdots a_{q}$. Now the relations in (2) imply

(3) if $1 \leqslant k \leqslant p$ and $n p+k \leqslant p+q$ then $a_{n p+1} \cdots a_{n p+k}=$ $b^{n} a_{1} \cdots a_{k} b^{-n}$. Define $x_{k}=a_{k} \cdots a_{k+q-1}$ for $1 \leqslant k \leqslant p+1$. Then the relations in (1) are $x_{1}=x_{2}=\cdots=x_{p+1}$. We wish to write each $x_{k}$ in terms of $a_{1}, \cdots, a_{p}$ and $b$. From this point, we proceed in two cases.

Case 1. $p<q$. Let $q=j p+\delta$ where $0<\delta<p$. Using the relations in (3),

$$
\begin{aligned}
x & =x_{1}=a_{1} \cdots a_{q} \\
& =\left(a_{1} \cdots a_{p}\right)\left(a_{p+1} \cdots a_{2 p}\right) \cdots\left(a_{(j-1) p+1} \cdots a_{j p}\right)\left(a_{j p+1} \cdots a_{j p+\delta}\right) \\
& =\left(a_{1} \cdots a_{p}\right)\left(b a_{1} \cdots a_{p} b^{-1}\right) \cdots\left(b^{j-1} a_{1} \cdots a_{p} b^{1-j}\right)\left(b^{j} a_{1} \cdots a_{\delta} b^{-j}\right) \\
& =\left(a_{1} \cdots a_{p} b\right)^{j}\left(a_{1} \cdots a_{\delta}\right) b^{-j} .
\end{aligned}
$$

Similarly,

$$
\begin{aligned}
x_{2} & =a_{2} \cdots a_{q+1} \\
& =\left(a_{2} \cdots a_{p}\right)\left(a_{p+1} \cdots a_{2 p}\right) \cdots\left(a_{(j-1) p+1} \cdots a_{j p}\right)\left(a_{j p+1} \cdots a_{j p+\delta+1}\right) \\
& =\left(a_{2} \cdots a_{p} b\right)\left(a_{1} \cdots a_{p} b\right)^{j-1}\left(a_{1} \cdots a_{\delta+1}\right) b^{-j} .
\end{aligned}
$$

In general, for $1 \leqslant k \leqslant p$,

$$
\begin{aligned}
x_{k}=\left(a_{k} \cdots a_{p} b\right)\left(a_{1} \cdots a_{p} b\right)^{j-1}\left(a_{1} \cdots a_{\delta+k-1}\right) b^{-j} \\
\text { if } \delta+k-1 \leqslant p, \text { or }
\end{aligned}
$$

$$
\begin{aligned}
& x_{k}=\left(a_{k} \cdots a_{p} b\right)\left(a_{1} \cdots a_{p} b\right)^{j}\left(a_{1} \cdots a_{\delta+k-1-p}\right) b^{-j-1} \text { if } \delta+k-1>p ; \\
& x_{p+1}=b\left(a_{1} \cdots a_{p} b\right)^{j}\left(a_{1} \cdots a_{\delta-1}\right) b^{-j-1} .
\end{aligned}
$$

Now we are ready to find $a_{1}$, a meridian of $K(p, q)$, in terms of $x, y$ and $b$. We write $x^{\beta}=z_{1} z_{2} \cdots z_{\beta}$ where each $z_{i}=a_{1} \cdots a_{q}$ and we make a replacement for each $z_{i}$ to find a new representation for $x^{\beta}$. Replace $z_{1}$ by

$$
v_{1}=x_{1}=\left(a_{1} \cdots a_{p} b\right)^{j}\left(a_{1} \cdots a_{\delta}\right) b^{-j} \text {. }
$$

Replace $z_{2}$ by $v_{2}=x_{\delta+1}$ where $x_{\delta+1}$ is written as in (4). Note that the last " $a$ " letter in $v_{1}$ is $a_{\delta}$ and the first " $a$ " letter in $x_{\delta+1}$ is $a_{\delta+1}$. In general, 
(a) if $z_{i}$ is replaced by

$$
v_{i}=x_{k}=a_{k} \cdots a_{p} b\left(a_{1} \cdots a_{p} b\right)^{j-1}\left(a_{1} \cdots a_{\delta+k-1}\right) b^{-j}
$$

then $z_{i+1}$ is replaced by $x_{\delta+k}$,

(b) if $z_{i}$ is replaced by

$$
v_{i}=a_{k} \cdots a_{p} b\left(a_{1} \cdots a_{p} b\right)^{j}\left(a_{1} \cdots a_{\delta+k-1-p}\right) b^{-j-1}
$$

then $z_{i+1}$ is replaced by $v_{i+1}=x_{\delta+k-p}$, and

(c) if $z_{i}$ is replaced by

$$
v_{i}=b\left(a_{1} \cdots a_{p} b\right)^{j}\left(a_{1} \cdots a_{\delta-1}\right) b^{-j-1}
$$

then $z_{i+1}$ is replaced by $v_{i+1}=x_{\delta}$, where each of the $x_{l}$ 's is written as in (4). Note that the $v_{i}$ terms are chosen so that the " $a$ " terms "match up," that is, if the last " $a$ " letter in $v_{i}$ is $a_{l}$ then the first " $a$ " letter in $v_{i+1}$ is $a_{l+1}$. A general formula is $v_{i}=x_{[(i-1) q]+1}$ where, for $m$ an integer, $[m]$ is defined as follows:

$$
\begin{array}{ll}
{[m]=\mu} & \text { if } m=\sigma p+\mu, 0<\mu<p, \\
{[m]=p} & \text { if } m=o p .
\end{array}
$$

Let us examine the product $v_{1} v_{2} \cdots v_{\beta}$. If all the $b$ 's were deleted it would be

$$
\left(a_{1} \cdots a_{p}\right)\left(a_{1} \cdots a_{p}\right) \cdots\left(a_{1} \cdots a_{p}\right) a_{1}
$$

with the group $\left(a_{1} \cdots a_{p}\right)$ appearing $(-\alpha)$ times. The last letter is $a_{1}$ since the product $x^{\beta}=\left(a_{1} \cdots a_{q}\right)^{\beta}$ contains $\beta q$ " $a$ " letters which we have divided into $(-\alpha)$ groups of $p$ plus one. Now let us consider where the various $b$ terms lie in $v_{1} \cdots v_{\beta}$. First, there is a $b$ (with no exponent) after each $a_{p}$. Also, there are the $b^{-j}$ and $b^{-j-1}$ terms. Now $b$ and $x$ commute, $v_{i}=x$ in $\pi_{1}(T-K(p, q))$, and the terms $b^{-j}$ and $b^{-j-1}$ appear at the end of each $v_{i}$. Hence, all terms of this form may be commuted to the right-hand end of the product. Hence,

$$
x^{\beta}=v_{1} v_{2} \cdots v_{\beta}=\left(a_{1} \cdots a_{p} b\right)^{-\alpha} a_{1} b^{\mu}
$$

where $b^{\mu}$ is the product of all the terms of the form $b^{-j}$ or $b^{-j-1}$.

Next we show $\mu=-\alpha$. Now $x^{\beta}$ is homologous in $T-K(p, q)$ to $a_{1}^{\beta q}=$ $a_{1}^{1-\alpha p}$. But if we look at $x^{\beta}=\left(a_{1} \cdots a_{p} b\right)^{-\alpha} a_{1} b^{\mu}$ as a word in $H_{1}(T-K(p, q))$ we get $x^{\beta}=a_{1}^{-\alpha p+1} b^{-\alpha+\mu}$; hence $-\alpha+\mu=0$. Therefore,

$$
x^{\beta}=\left(a_{1} \cdots a_{p} b\right)^{-\alpha} a_{1} b^{\alpha}=y^{-\alpha} a_{1} b^{\alpha} .
$$


Hence, $a_{1}=y^{\alpha} x^{\beta} b^{-\alpha}$. Therefore, in case $p<q$, a meridian of $K(p, q)$ is $y^{\alpha} x^{\beta} b^{-\alpha}$. and $b$ :

Case 2. $p>q$. As in Case 1, we write each $x_{k}$ in terms of $a_{1}, \cdots, a_{p}$,

$$
\begin{aligned}
x_{k} & =a_{k} \cdots a_{k+q-1} \quad \text { if } k+q-1 \leqslant p, \\
x_{k} & =a_{k} \cdots a_{p} b a_{1} \cdots a_{k+q-1-p} b^{-1} \text { if } p<k+q-1<p+q ; \\
x_{p+1} & =b a_{1} \cdots a_{q} b^{-1} .
\end{aligned}
$$

As in Case 1, we write $x^{\beta}=z_{1} \cdots z_{\beta}$ where each $z_{i}=a_{1} \cdots a_{q}$. Again we replace each $z_{i}$ in this product. $z_{1}$ is replaced by $v_{1}=x_{1}=a_{1} \cdots a_{q}$. In general,

(a) if $z_{i}$ is replaced by $v_{i}=x_{k}=a_{k} \cdots a_{k+q-1}$ with $k+q-1 \leqslant p$ then $z_{i+1}$ is replaced by $v_{i+1}=x_{k+q}$, written as in (5),

(b) if $z_{i}$ is replaced by $v_{i}=a_{k} \cdots a_{p} b a_{1} \cdots a_{k+q-1-p} b^{-1}$ then $z_{i+1}$ is replaced by $v_{i+1}=x_{k+q-p}$, and

(c) if $z_{i}$ is replaced by $v_{i}=x_{p+1}=b a_{1} \cdots a_{q} b^{-1}$ then $z_{i+1}$ is replaced by $v_{i+1}=x_{q+1}$.

Then $x^{\beta}=v_{1} v_{2} \cdots v_{\beta}$ and if all the $b$ 's were removed, the product would be $\left(a_{1} \cdots a_{p}\right)^{-\alpha} a_{1}$. Now there is a $b$ after every $a_{p}$ and there is a $b^{-1}$ at the end of each $v_{i}$. As in Case 1 , the $b^{-1}$ terms may be commuted to the right-hand end of the product and we have

$$
x^{\beta}=\left(a_{1} \cdots a_{p} b\right)^{-\alpha} a_{1} b^{\alpha}=y^{-\alpha} a_{1} b^{\alpha} .
$$

Hence, in either case, a meridian of $K(p, q)$ is $c=a_{1}=y^{\alpha} x^{\beta} b^{-\alpha}$. Now $x^{p} b^{q}=y^{q}$ is a longitude of $K(p, q)$ and $y^{q} \sim a_{1}^{p q} b^{q}$ in $T-K(p, q)$. Let $g=c^{-p q} y^{q}$. Then $g \sim b^{q}$, so $g$ is homologous, in $T-K(p, q)$, to a multiple of $L$.

Now $G(K: r, s)$ is obtained from $\pi_{1}(T-K(p, q))$ by adding the relation $c^{r} g^{s}=1$, that is, $c^{r}\left(c^{-p q} y^{q}\right)^{s}=1$. Now $c$ is a meridian of $K$ and $y^{q}$ is a longitude; hence they commute in $\pi_{1}(T-K(p, q))$. Therefore, the added relation is $c^{r-s p q} y^{s q}=1$ or $\left(y^{\alpha} x^{\beta} b^{-\alpha}\right)^{r-s p q} y^{q s}=1$. In this lemma we are assuming $r-s p q=1$; hence $G(K: r, s)$ has the following presentation:

$$
\left\{b, x, y: b x=x b, y^{q}=b^{q} x^{p}, y^{\alpha} x^{\beta} b^{-\alpha} y^{q s}=1\right\} .
$$

Our next step is to eliminate $y$ from this presentation.

The last relation is $y^{\alpha}=y^{-q s} b^{\alpha} x^{-\beta}$ or

$$
y^{\alpha}=\left(y^{q}\right)^{-s} b^{\alpha} x^{-\beta}=\left(b^{q} x^{p}\right)^{-s} b^{\alpha} x^{-\beta}=b^{\alpha-s p} x^{-s p-\beta} \text {. }
$$


Hence, $y=y^{\alpha p+\beta q}=\left(y^{\alpha}\right)^{p}\left(y^{q}\right)^{\beta}=\left(b^{\alpha-s q} x^{-s p-\beta}\right)^{p}\left(b^{q} x^{p}\right)^{\beta}=b^{1-s p q} x^{-s p^{2}}$.

Substituting for $y$ in the second relation in (6), we obtain

$$
\left(b^{1-s p q} x^{-s p^{2}}\right)^{q}=b^{q} x^{p} \quad \text { or } \quad b^{-s p q^{2}}=x^{p(1+s p q)}=x^{p r} \text {. }
$$

Substituting for $y$ in $y^{\alpha}=b^{\alpha-s q} x^{-s p-\beta}$, which is the same as the last relation in (4), we obtain $\left(b^{1-s p q} x^{s p^{2}}\right)^{\alpha}=b^{\alpha-s p} x^{-s p-\beta}$, which reduces to

$$
\begin{aligned}
x^{\beta+s p(1-\alpha p)} & =b^{s q(\alpha p-1)}, \text { or } \\
x^{\beta+\beta s p q} & =b^{-\beta s q^{2}}, \text { or } \\
x^{\beta r} & =b^{-\beta s q^{2}} .
\end{aligned}
$$

Hence $G(K: r, s)$ is isomorphic to

$$
\left\{b, x: b x=x b, x^{p r}=b^{-s p q^{2}}, x^{\beta r}=b^{-\beta s q^{2}}\right\} .
$$

The last two relations imply

$$
x^{r}=\left(x^{p \eta}\right)^{\alpha}\left(x^{\beta \eta}\right)^{q}=b^{-\alpha s p q^{2}-\beta s q^{3}}=b^{-s q^{2}(\alpha p+\beta q)}=b^{-s q^{2}} .
$$

But the relation $x^{r}=b^{-s q^{2}}$ implies the last two relations in (5). Hence the group in (5) is isomorphic to $\left\{b, x: b x=x b, x^{r} b^{s q^{2}}=1\right\}$.

Now if $C$ is a simple closed curve on $\mathrm{Bd} T$ which is homologous (on Bd $T$ ) to $r M+s q^{2} L$ then $C$ is homotopically nontrivial on $\mathrm{Bd} T$ but the element of $G(K: r, s)$ generated by $C$ is $x^{r} b^{s q^{2}}$. Hence $C$ is null-homotopic in $T(K: r, s)$ so $C$ is a meridian of $T(K: r, s)$.

LEMMA 2(b). If $r=1$ then

$$
G_{d}(K(p, q): r, s)=\left\{b, x: b x=x b, x^{r+s p q} b^{s q^{2}}=1\right\}
$$

and a curve homologous to $(r+s p q) M+s q^{2} L$ is a meridian of the solid torus $T_{d}(K: r, s)$, where $x$ and $b$ are as in Lemma 2(a). If $r=-1$ then the conclusion is the same with $s$ replaced by $-s$.

This lemma follows from Lemma 2(a) and the remarks which precede Corollary 1.

Corollary 5. If $K$ is $a(p, q)$ torus knot in $S^{3}$ and $|r-s p q|=1$ then $M^{3}(K: r, s)$ is an $L\left(s q^{2}, r\right)$ lens space.

Proof. From [1, p. 108], a lens space $L\left(s q^{2}, r\right)$ is homeomorphic to a union of two solid tori $T_{1}$ and $T_{2}$ where $T_{1} \cap T_{2}=\mathrm{Bd} T_{1}=\mathrm{Bd} T_{2}$ and such that a meridian of $T_{1}$ is sewn to a curve $C$ on $\mathrm{Bd} T_{2}$ with $C \sim s q^{2} M_{2}+$ $r L_{2}$ where $\left(M_{2}, L_{2}\right)$ is a meridian-longitude pair for $T_{2}$.

Now the manifold $M^{3}(K: r, s)$ is obtained from $S^{3}$ by removing the 
interior of a regular neighborhood $T^{\prime}$ of $K$ and sewing in a solid torus $T^{\prime \prime}$ such that a meridian of $T^{\prime \prime}$ is sewn to a curve on $\mathrm{Bd} T^{\prime}$ homologous to $r c+$ $s q$ where $(c, g)$ is a meridian-longitude pair for $T^{\prime}$ with $g \sim 0$ in $S^{3}-K$. Let $T$ be an unknotted solid torus in $S^{3}$ such that $K \subset$ Int $T$ and $K$ cobounds an annulus with a curve $K_{1}$ on $\mathrm{Bd} T$ such that $K_{1} \sim r c+s g$ on $\mathrm{Bd} T$. Let $T_{2}=S^{3}$ - Int $T$. Then the embedding of $K$ in $T$ is of the kind considered in Theorem 2; hence $M^{3}(K: r, s)=T(K: r, s) \cup T_{2}$. Since $|r-s p q|=1$, $T(K: r, s)$ is a solid torus. Also, by Lemma 2(a), a meridian of $T(K: r, s)$ is a curve on $\mathrm{Bd} T$ homologous to $r M \pm s q^{2} L$ where $(M, L)$ is a meridian-longitude pair for $T$ such that $(L, M)$ is a meridian-longitude pair for $T_{2}$. Therefore, by the remarks of the preceding paragraph, $M^{3}(K: r, s)$ is the lens space $L\left(s q^{2}, r\right)$.

We conclude this section with two remarks concerning some results of $J$. Simon [7].

REMARK 1. In Theorem 3 of [7], Simon noted the following: If $K$ is a knot in $S^{3}$ and $J$ is a $(1,2)$ cable about $K$ then $\pi_{1}\left(M^{3}(J: 1,1)\right) \cong$ $\pi_{1}\left(M^{3}(K: 1,4)\right)$.

The results of this section may be used to conclude the following: If $T$ is a regular neighborhood of $K$ in $S^{3}$ such that $J \subset$ Int $T$ and $J$ cobounds an annulus with a $(1,2)$ curve on $\mathrm{Bd} T$, then

$$
M^{3}(J: 1,1)=T(J(1,1)) \cup\left(S^{3}-\text { Int } T\right) .
$$

Now in this case, $r-s p q=-1$; hence $T(J: 1,1)$ is a solid torus by Corollary 3. By Lemma 2, a meridian of $T(J: 1,1)$ is a curve on $\operatorname{Bd} T$ homologous to $M+4 L$ where $(M, L)$ is a meridian-longitude pair for $T$ such that $L \sim 0$ in $S^{3}$ - Int $T$. Therefore, we may conclude that the two fundamental groups mentioned above are isomorphic because the two surgery manifolds $M^{3}(J: 1,1)$ and $M^{3}(K: 1,4)$ are homeomorphic.

REMARK 2. In Theorem 4 of [7], Simon noted that a simply connected 3manifold can be obtained by nontrivial surgery on a link $K \cup J$ in $S^{3}$ where $K$ is a nontrivial knot and $J$ is a cable about $K$. The results of this section can be used to conclude two things in this regard: (1) Any homotopy 3-sphere obtained by this kind of surgery can also be obtained by surgery on $K$, and (2) $S^{3}$ can be obtained by nontrivial surgery on such a link.

First, we describe the construction in detail. Let $K$ be a knot in $S^{3}$ and suppose $J$ is a $(p, q)$ cable about $K$. This means there is a solid torus $T^{*}$ in $S^{3}$ with a meridian-longitude pair $\left(M^{*}, L^{*}\right)$ such that:

(i) there is a simple closed curve $J^{*}$ in Int $T^{*}$ which cobounds an annulus with a curve $J_{1}^{*}$ on $\mathrm{Bd} T^{*}$ such that $J_{1}^{*} \sim p M^{*}+q L^{*}$, and 
(ii) there is a homeomorphism $f$ of $T^{*}$ into $S^{3}$ such that $f$ takes a core of $T^{*}$ onto $K, f\left(J^{*}\right)=J$ and $f\left(L^{*}\right) \sim 0$ in $S^{3}-\operatorname{Int} f\left(T^{*}\right)$.

Let $T=f\left(T^{*}\right), M=f\left(M^{*}\right), L=f\left(L^{*}\right)$ and $J_{1}=f\left(J_{1}^{*}\right)$. Let $T_{K}$ be a regular neighborhood of $K$ in Int $T$ such that $T_{K} \cap J=\varnothing$. Let $\left(c_{K}, g_{K}\right)$ be a meridian-longitude pair for $T_{K}$ such that $g_{K} \sim f(L)$ in $T-K$. Let $T_{J}$ be a regular neighborhood of $J$ in Int $T$ such that $T_{J} \cap T_{K}=\varnothing$. Let $\left(c_{J}, g_{J}\right)$ be a meridian-longitude pair for $T_{J}$ such that $g_{J} \sim J_{1}$ in $T-(K \cup J)$.

Now suppose $\left(r_{1}, s_{1}\right)$ and $\left(r_{2}, s_{2}\right)$ are pairs of relatively prime integers. Let $M^{3}=M^{3}\left(K: r_{1}, s_{1} \mid V: r_{2}, s_{2}\right)$ denote the manifold obtained by $\left(r_{1}, s_{1}\right)$ surgery on $K$ and $\left(r_{2}, s_{2}\right)$-surgery on $J$. That is $M^{3}$ is obtained from $S^{3}$ by removing $T_{K}$ and $T_{J}$ and sewing in solid tori $T_{K}^{\prime \prime}$ and $T_{J}^{\prime \prime}$ where a meridian of $T_{K}^{\prime \prime}$ is sewn to a curve homologous to $r_{1} c_{K}+s_{1} g_{K}$ and a meridian of $T_{J}$ is sewn to a curve homologous to $r_{2} c_{J}+s_{2} g_{J}$. We consider the surgery on $K$ first. Let $T_{1}=\left(T-\operatorname{Int} T_{K}\right) \cup T_{K}^{\prime \prime} . T_{1}$ is a solid torus since it is a union of a solid torus, namely $T_{K}^{\prime \prime}$, and a,boundary collar. Also a curve $M_{1}$ on $\operatorname{Bd} T$ with $M_{1} \sim r_{1} M+s_{1} L$ is a meridian of $T_{1}$ and $J$ is a nicely embedded torus knot in $T_{1}$.

Now $r_{1}$ and $s_{1}$ are relatively prime; hence there are integers $\alpha$ and $\beta$ such that $\alpha r_{1}+\beta s_{1}=1$. Let $L_{1}$ be a simple closed curve on $\operatorname{Bd} T_{1}=\operatorname{Bd} T$ such that $L_{1} \sim \beta M-\alpha L$. We claim that $L_{1}$ is a longitude of $T_{1}$. First, $M \sim$ $\alpha M_{1}+s_{1} L_{1}$ and $L \sim \beta M_{1}-r_{1} L_{1}$ so $M_{1}$ and $L_{1}$ generate the homology group $H_{1}\left(\mathrm{Bd} T_{1}\right)$. It remains to show that the algebraic intersection number $\#\left(M_{1}, L_{1}\right)= \pm 1$. But $\#\left(M, M_{1}\right)=s_{1}$ and $\#\left(L, M_{1}\right)=-r_{1}$; hence $\#\left(L_{1}, M_{1}\right)=$ $\#\left(\beta M-\alpha L, M_{1}\right)=\beta s_{1}-\alpha\left(-r_{1}\right)=1$. Hence $\left(M_{1}, L_{1}\right)$ is a meridian-longitude pair for the solid torus $T_{1}$.

Now, $J_{1} \sim p M+q L \sim(\alpha p+\beta q) M_{1}+\left(p s_{1}-q r_{1}\right) L_{1}$; hence $J$ is an $\left(\alpha p+\beta q, p s_{1}-q r_{1}\right)$ torus knot in $T$. Let $p^{\prime}=\alpha p+\beta q$ and $q^{\prime}=p s_{1}-q r_{1}$. Let $T_{2}$ denote the result of $\left(r_{2}, s_{2}\right)$ on the knot $J$ in $T_{1}$. That is, $T_{2}=$ $\left(T_{1}-\right.$ Int $\left.T_{J}\right) \cup T_{J}^{\prime \prime}$.

Case 1. If $r_{2} \neq \pm 1$, then $M^{3}$ is not a homotopy 3 -sphere. If $M^{3}$ were simply connected then, by Dehn's lemma, once closed complementary domain of the torus $\mathrm{Bd} T$ would be a homotopy solid torus. But one complementary domain is $S^{3}$ - Int $T$ and the other is $T_{2}$, neither of which is a homotopy solid torus.

Case 2. $r_{2}= \pm 1$. Then $T_{2}$ is a solid torus and, by Lemma 2(b), a meridian of $T_{2}$ is a curve $M_{2}$ on $\mathrm{Bd} T=\mathrm{Bd} T_{2}$ such that $M_{2} \sim\left(r_{2}+s_{2} p^{\prime} q^{\prime}\right) M_{1}$ $+s_{2}\left(q^{\prime}\right)^{2} L_{1}$. Letting $p^{\prime}=\alpha p+\beta q, q^{\prime}=p s_{1}-q r_{1}, \quad M_{1}=r_{1} M+s_{1} L$ and $L_{1}=\beta M-\alpha L$ we obtain, using the equation $\alpha r_{1}+\beta s_{1}=1$,

$$
M_{2} \sim\left(r_{1} r_{2}+p^{2} s_{1} s_{2}-p q r_{1} s_{2}\right) M+\left(r_{2} s_{1}+p q s_{1} s_{2}-q^{2} r_{1} s_{2}\right) L
$$


Therefore, if $r_{2}= \pm 1$, the surgery manifold $M^{3}$ is homeomorphic to $M^{3}\left(K: r_{1} r_{2}+p^{2} s_{1} s_{2}-p q r s_{1} s_{2}, r_{2} s_{1}+p q s_{1} s_{2}-q^{2} r_{1} s_{2}\right)$.

Hence conclusion (1) follows from Case 1 and Case 2. Also it follows from Case 2 that $M^{3} \approx S^{3}$ if

$$
\begin{aligned}
r_{2} & = \pm 1, \\
r_{1} r_{2}+p^{2} s_{1} s_{2}-p q r_{1} s_{2} & = \pm 1, \quad \text { and } \\
r_{2} s_{1}+p q s_{1} s_{2}-q^{2} r_{1} s_{2} & =0 .
\end{aligned}
$$

4. Doubly twisted knots. Before defining doubly twisted knots and considering surgery on them, we describe a method of presenting the group of knots "with twists." Suppose $C$ is the cube $[0,1] \times[0,1] \times[0,1], A$ and $B$ are parallel rectilinear spanning segments of $C$ and $T$ is an unknotted solid torus in $C-(A \cup B)$. See Figure 2a. Suppose $T$ has a longitude $g$ which bounds a disk $D$ in $C$-Int $T$ such that $D$ intersects each of $A$ and $B$ in a single interior point. Let $c$ be a meridian of $T$. Suppose Int $T$ is removed from $C$ and a solid torus $T_{1}$ is sewn in with a meridian of $T_{1}$ sewn to a simple closed curve homologous to $c+n g$. Then there is a homeomorphism of $(C-$ Int $T) \cup T_{1}$ onto $C$ which is the identity on $\mathrm{Bd} C$ and which takes $A$ and $B$ onto arcs $A^{\prime}$ and $B^{\prime}$, as in Figure $2 b$, where $A^{\prime}$ and $B^{\prime}$ have $2 n$ crossings. We construct this homeomorphism as follows:

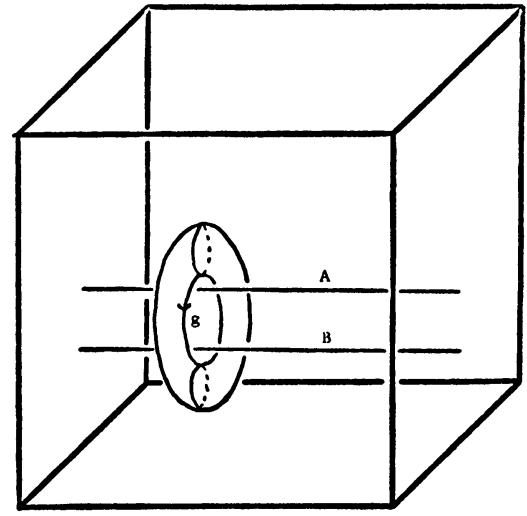

FIGURE 2a

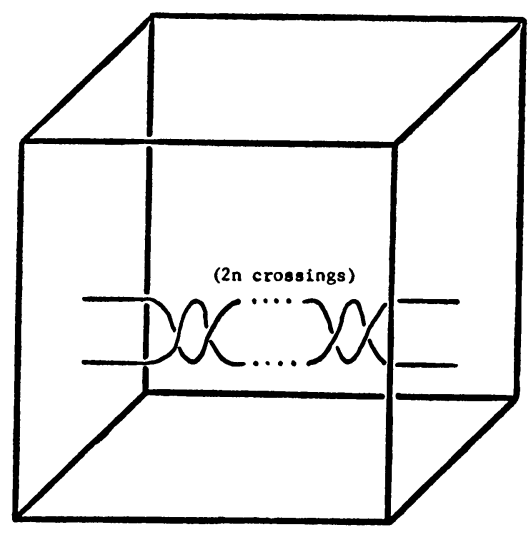

FIGURE $2 b$

Let $h$ be a homeomorphism of $D \times[-1,1]$ into $C$-Int $T$ such that $h(D \times\{0\})=D$ and $h(\mathrm{Bd} D \times[-1,1]) \subset \mathrm{Bd} T=\mathrm{Bd} T_{1}$. Let $f$ be a homeomorphism of $h(D \times[-1,1])$ onto itself which is the identity on $D \times$ $\{-1,1\}$ and which gives one end of $h(D \times[-1,1]) n$ full twists in the $+g$ direction. Let $f$ be the identity on $C-(h(D \times[-1,1]) \cup$ Int $T)$. Since $f$ 
takes a meridian of $T$ onto a curve homologous to $c+n g, f$ may be extended to take $C$ onto $(C-\operatorname{Int} T) \cup T_{1}$. Then $f^{-1}$ is the required homeomorphism. The action of $f^{-1}$ on the pair $(A, B)$ may be visualized as follows: $f^{-1}$ cuts the pair in the middle, gives them $n$ full twists in the $-g$ direction and pastes them back together.

The twisting homeomorphism $f^{-1}$ provides a connection between certain knot groups and groups obtained by adding a surgery relation to an appropriate link group. For example, the group of the $2 n$-twist knot, Figure 3a, may be presented as the group of the link $K \cup J$ in Figure $3 \mathrm{~b}$ with the added relation $a\left(y^{-1} x\right)^{-n}=1$.

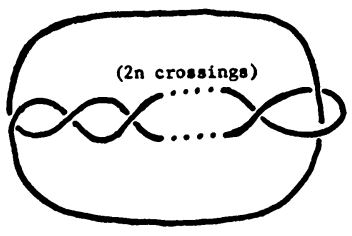

FIGURE 3a

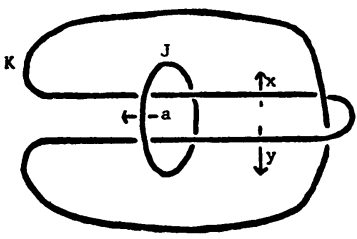

FIGURE 3b

In the remainder of this section, $T(m, n)$ will denote the doubly twisted knot in the solid torus $T$ as shown in Figure 4. Here $n \neq 0$, but $m$ is any integer. Also, if $n$ is odd then $m$ must be even, since otherwise we would have two curves.

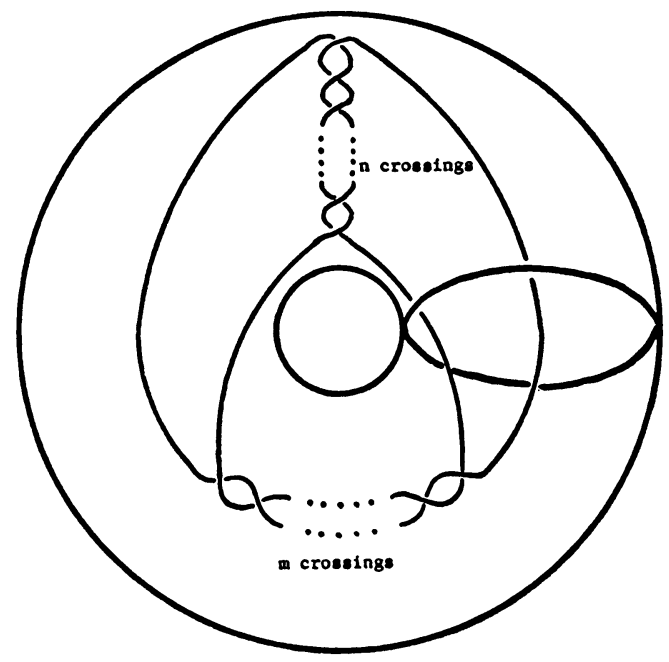

FIGURE 4

Theorem 3. For $(m, n) \neq($ even, \pm 1$), T(T(m, n): r, s)$ is a homotopy solid torus only in the trivial case $(r, s)=( \pm 1,0)$. 
Note that in case $(m, n)=($ even, \pm 1$)$ the $\operatorname{knot} T(m, n)$ is a torus knot as considered in $\S 3$.

Proof of Theorem 3. We consider three cases: (1) $(m, n)=$ (even, even), (2) $(m, n)=$ (odd, even), and (3) $(m, n)=$ (even, odd) with $n \neq \pm 1$. In each case we show that the fundamental group of $T(T(m, n): r, s)$ is not infinite cyclic.

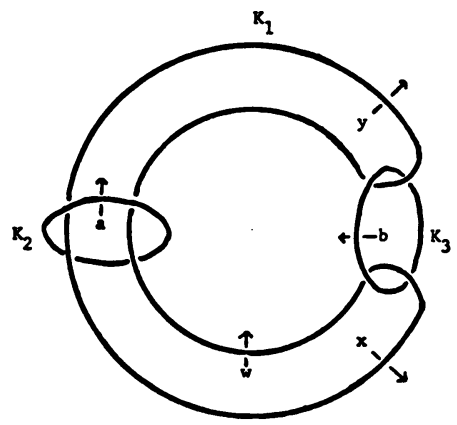

FIGURE 5

Case 1. Both $m$ and $n$ are even. For a fixed even integer $n$, the manifolds $T(m, n), m$ even, are equivalently embedded in $T$. Hence we consider only the case $m=0$. To calculate $\pi_{1}(T-T(m, n))$ we consider the link in Figure 5. The group $\pi_{1}(T-T(m, n))$ will be calculated as $\pi_{1}\left(T-\left(K_{1} \cup K_{2} \cup K_{3}\right)\right)$ with the added relation $b\left(y x^{-1}\right)^{-k}=1$, where $n=2 k$. The added relation comes from surgery on $K_{3}$ and gives the knot the $2 k$ crossings. Hence $\pi_{1}(T-T(0, n))$ has a presentation with generators $a, b, w, x$ and $y$ and relations:
(1) $a^{-1} w a=b^{-1} y b$,
(2) $x a x^{-1}=w a w^{-1}$,
(3) $y^{-1} b y=x^{-1} b x$,
(4) $y=a^{-1} x a$,
(5) $w=b^{-1} x b$, and
(6) $b=\left(y x^{-1}\right)^{k}$.

Now (3) is a consequence of (6). We add a generator $z$ and the relation $z=$ $y x^{-1}$ and then we eliminate $y$. Then, using (6), we eliminate $b$. We obtain a presentation with generators $a, w, x$ and $z$ and relations:
(1') $a^{-1} w a=z^{1-k} x z^{k}$,
(2') $x a x^{-1}=w a w^{-1}$,
(4') $z x=a^{-1} x a$,
(5') $w=z^{-k} x z^{k}$.

Now $\left(5^{\prime}\right),\left(4^{\prime}\right)$ and $\left(2^{\prime}\right)$ imply $\left(1^{\prime}\right)$; hence $\pi_{1}(T-T(0, n))$ has the presentation

$$
\left\{a, w, x, z: z=a^{-1} x a x^{-1}=a^{-1} w a w^{-1}, w=z^{-k} x z^{k}\right\} \text {. }
$$

Now a meridian of $K_{3}=T(0, n)$ is $c=w$ and a longitude is $g=a b^{-1} a^{-1} b=$ $a z^{-k} a^{-1} z^{k}$. Note that $g \sim 0$ in $T-T(0, n)$. Then the surgery manifold 
$T(T(0, n): r, s)$ has fundamental group $G(T(0, n): r, s)$ which is obtained from (7) by adding the relation $c^{r} g^{s}=1$ or $w^{r}\left(a z^{-k} a^{-1} z^{k}\right)^{s}=1$. If this presentation of $G(T(m, n): r, s)$ is abelianized, we obtain the group $\{a, w: a w=w a$, $w^{r}=1$ \} which is not infinite cyclic unless $r= \pm 1$. Hence we assume $r=-1$. (The case $r=1$ is essentially the same.) Hence we have the group

(8) $\left\{a, w, x, z: z=a^{-1} x a x^{-1}=a^{-1} w a w^{-1}, w=z^{-k} x z^{k}, w=\left(a z^{-k} a^{-1} z^{k}\right)^{s}\right\}$.

We eliminate $w$ using the third relation and obtain relations

$$
\begin{aligned}
z & =a^{-1} x a x^{-1}, \\
z & =a^{-1} z^{-k} x z^{k} a z^{-k} x^{-1} z^{k}, \text { and } \\
z^{-k} x z^{k} & =\left(a z^{-k} a^{-1} z^{k}\right)^{s} \text { or } x=\left(z^{k} a z^{-k} a^{-1}\right)^{s} .
\end{aligned}
$$

Using (11), we eliminate $x$ and obtain:

$$
z=a^{-1}\left(z^{k} a z^{-k} a^{-1}\right)^{s} a\left(z^{k} a z^{-k} a^{-1}\right)^{-s}=\left(a^{-1} z^{k} a z^{-k}\right)^{s}\left(z^{k} a z^{-k} a^{-1}\right)^{-s},
$$

and

$$
\begin{aligned}
& z=\left(z^{-k} a^{-1} z^{k} a\right)^{s}\left(a z^{-k} a^{-1} z^{k}\right)^{-s} \text { or } \\
& z=z^{k}\left(z^{-k} a^{-1} z^{k} a\right)^{s} z^{-k} z^{k}\left(a z^{-k} a^{-1} z^{k}\right)^{-s} z^{-k} \text { or } \\
& z=\left(a^{-1} z^{k} a z^{-k}\right)^{s}\left(z^{k} a z^{-k} a^{-1}\right)^{-s} .
\end{aligned}
$$

These calculations show $\left(9^{\prime}\right)$ and $\left(10^{\prime}\right)$ are the same; hence $G(T(0, n):-1, s)$ has the presentation

$$
\left\{a, z: z=\left(a^{-1} z^{k} a z^{-k}\right)^{s}\left(z^{k} a z^{-k} a^{-1}\right)^{-s}\right\} .
$$

We obtain a quotient group by adding the relation $a^{2}=1$. The quotient group is $\left\{a, z: a^{2}=1, z=\left(a z^{k} a z^{-k}\right)^{2 s}\right\}$. The second relation is $z^{2 k s+1}=\left(a z^{k} a\right)^{2 s}=$ $a z^{2 k s} a$ or $a z^{2 k s+1}=z^{2 k s} a$. Hence the quotient group is

$$
\left\{a, z: a^{2}=1, a z^{2 k s+1}=z^{2 k s} a\right\} \text {. }
$$

Now the relations in (12) imply $z^{2 k s+1} a z=a z^{2 k s+1}$, or $z^{2 k s+1} a z=z^{2 k s} a$, or $z a z=a$, or $(a z)^{2}=1$. The relations in (12) and this last relation imply $a z a=z^{-1}$ which implies $a z^{2 k s} a=z^{-2 k s}$. Using the relations in (12), we change this to $a z^{2 k s} a=a z^{-2 k s-1} a$ or $z^{4 k s+1}=1$. Hence the relations in (12) imply $a^{2}=(a z)^{2}=z^{4 k s+1}=1$. Now these last relations imply $a z a=z^{-1}$ which implies $a z^{2 k s} a=z^{-2 k s}$, or $a z^{2 k s} a=z^{2 k s+1}$, or $a z^{2 k s}=z^{2 k s+1} a$. Hence, the group (12) is the same as $\left\{a, z: a^{2}=(a z)^{2}=z^{4 k s+1}=1\right\}$. But this is a presentation of the dihedral group $\mathfrak{D}_{4 k s+1}$. See $[5$, p. 6]. Since $|4 k s+1| \neq 1, G(T(0, n):-1, s)$ is nonabelian because it has the nonabelian quotient $\mathfrak{D}_{4 k s+1}$. 
Case 2. $m$ is odd and $n$ is even. Say $n=2 k$. As in Case 1 , for fixed $n$, the knots $T(m, n), m$ odd, are equivalently embedded in $T$. Hence we consider only the case $m=1$. We find a presentation for $\pi_{1}(T-T(1, n))$ by considering the link in Figure 6 and adding a relation corresponding to surgery on $K_{3}$. Hence we have a presentation with generators $a, b, w, x$ and $y$ and relations:
(13) $a^{-1} w a=b^{-1} y b$,
(14) $x a x^{-1}=w a w^{-1}$,
(15) $y^{-1} b y=w b w^{-1}$
(16) $x=a y a^{-1}$,
(17) $w^{-1} x w=b^{-1} w b$,
(18) $b(y w)^{-k}=1$.

Now (15) is a consequence of (18). We add a generator $z$ and the relation $z=$ $y w$ and then eliminate $y$. Next we eliminate $b$ using (18) and then $x$ using (16). We are left with generators $a, w$, and $z$ and the following relations:

$$
\begin{aligned}
a^{-1} w a & =z^{1-k} w^{-1} z^{k}, \\
a z w^{-1} a w z^{-1} a^{-1} & =w a w^{-1}, \quad \text { and } \\
w^{-1} a z w^{-1} a^{-1} w & =z^{-k} w z^{k} .
\end{aligned}
$$

Now $\left(17^{\prime}\right)$ is a consequence of $\left(13^{\prime}\right)$ and $\left(14^{\prime}\right)$; hence $\pi_{1}(T-T(1, n))$ has the presentation

$$
\left\{a, w, z: a^{-1} w a=z^{1-k} w^{-1} z^{k}, w a w^{-1}=a z w^{-1} a w z^{-1} a^{-1}\right\} .
$$

Now a meridian of $T(1, n)$ is $c=w$ and a natural longitude, from Figure 6, is $g_{1}=a b^{-1} a^{-1} w b^{-1}=a z^{-k} a^{-1} w z^{-k}$. Hence a longitude which is null-homologous in $T-T(1, n)$ is $g=w^{4 k-1} a z^{-k} a^{-1} w z^{-k}$. Therefore, $G(T(1, n): r, s)$

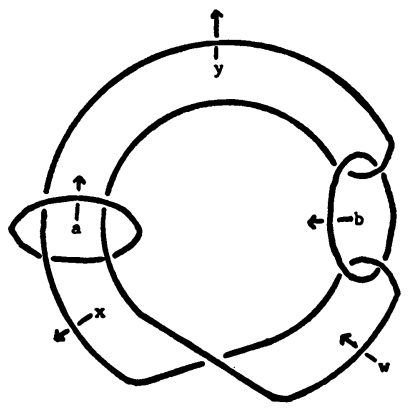

Figure 6

is found by adding to (19) the relation $c^{r} g^{s}=1$ or $w^{r+4 k s-s}\left(a z^{-k} a^{-1} w z^{-k}\right)^{s}$ $=1$. Now if $G(T(1, n): r, s)$ is abelianized, we obtain the group $\{a, w: a w=$ $\left.w a, w^{r}=1\right\}$; hence we may assume $|r|=1$. Since the two cases are essentially the same, we assume $r=1$. Then $G(T(1, n): 1, s)$ has the presentation 
(20)

$$
\begin{array}{r}
\left\{a, w, z: a^{-1} w a=z^{1-k} w^{-1} z^{k}, w a w^{-1}=a z w^{-1} a w z^{-1} a^{-1},\right. \\
\left.w^{4 k s-s+1}=\left(a z^{-k} a^{-1} w z^{-k}\right)^{s}\right\} .
\end{array}
$$

We obtain a quotient group by adding the relations $z^{k}=1$ and $a^{2}=1$. Then we use the first relation in (20) to eliminate $z$. This yields the group

$$
\left\{a, w: a^{2}=(a w)^{2 k}=w^{4 k s+1}=1\right\} .
$$

This group has the dihedral group $\mathfrak{D}_{4 k s+1}$ as a quotient; hence, as in Case 1, $G(T(1, n): r, s)$ is nonabelian.

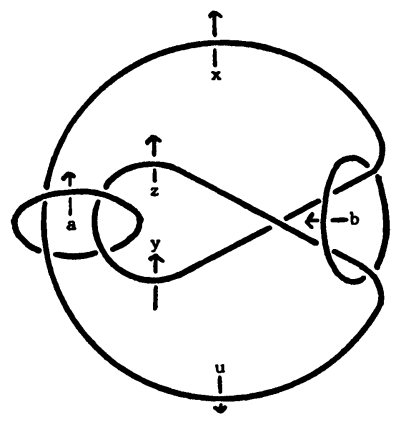

FiguRE 7

Case 3. $m$ is even, $n$ is odd and $n \neq \pm 1$. As in the first two cases, we consider only the case $m=0$ and we find a presentation for $\pi_{1}(T-T(0, n))$ by considering the link in Figure 7 and adding a surgery relation. Since a reflection of $T$ takes $T(0,-n)$ onto $T(0, n)$, we assume $n>0$. Hence we have a presentation with generators $a, b, u, x, y$ and $z$ and relations

(22) $u a u^{-1}=y^{-1} a y$,

(23) $x=a^{-1} u a$, (24) $y=a z a^{-1}$,

(25) $b^{-1} x b=z^{-1} y z$,

(26) $z=b^{-1} u b$, (27) $x^{-1} b x=u^{-1} b u$, and

(28) $b\left(x u^{-1}\right)^{-k}=1$

where $n=2 k+1$ (hence $k>0$ ). Now (27) is a consequence of (28). We add a new generator $t$ with $t=x u^{-1}$ and then eliminate $x$. Then, using (28), we eliminate $b$, and using (24), we eliminate $y$. We are left with the following relations:

$$
\begin{aligned}
u a u^{-1} & =a z^{-1} a z a^{-1}, \\
t & =a^{-1} u a u^{-1}, \\
t^{1-k} u t^{k} & =z^{-1} a z a^{-1} z, \text { and } \\
z & =t^{-k} u t^{k} .
\end{aligned}
$$

Now $\left(25^{\prime}\right)$ is a consequence of $\left(22^{\prime}\right),\left(23^{\prime}\right)$ and $\left(26^{\prime}\right)$. Also, we may eliminate 
$z$ using (26). We are left with generators $a, u$, and $t$ and relations

$$
\begin{aligned}
& u a u^{-1} a t^{-k} u^{-1} t^{k} a t^{-k} u t^{k} a^{-1}, \text { and } \\
& t=a^{-1} u a u^{-1} \text {. }
\end{aligned}
$$

Now, from Figure 7, a meridian of $T(0, n)$ is $c=u$ and a longitude is $g=$ $u a b z^{-1} a b^{-1}=u a u^{-1} t^{k} a t^{-k}$. Note that $g \sim a^{2}$, and $a$ is a longitude of the solid torus $T$. Hence, $G(T(0, n): r, s)$ has the presentation

$$
\begin{aligned}
& \left\{a, t, u: u a u^{-1}=a t^{-k} u^{-1} t^{k} a t^{-k} u t^{k} a^{-1}, t=a^{-1} u a u^{-1},\right. \\
& \left.u^{r+s}\left(a u^{-1} t^{k} a t^{-k}\right)^{s}=1\right\} .
\end{aligned}
$$

We now obtain some quotient groups. Adding the relation $t^{k}=1$ and eliminating $t$ we have

$$
\left\{a u: u a u^{-1}=a u^{-1} a u a^{-1},\left(a^{-1} u a u^{-1}\right)^{k}=1, u^{r+s}\left(a u^{-1} a\right)^{s}=1\right\} .
$$

Now the first relation is $a^{-1} u a^{-1} u=u a^{-1} u a^{-1}$ which is the same as $\left(u a^{-1}\right)^{2} u=u\left(u a^{-1}\right)^{2}$. Using this, the third relation is $u^{r+s}=\left(a^{-1} u a^{-1}\right)^{s}$ or $u^{r+s}=\left[u^{-1}\left(u a^{-1}\right)^{2}\right]^{s}$ or $u^{r+2 s}=\left(u a^{-1}\right)^{2 s}$. Therefore, adding the relation $\left(u a^{-1}\right)^{2}=1$ to (30), we obtain the quotient group

$$
G_{1}=\left\{a, u:\left(u a^{-1}\right)^{2}=\left(a^{-1} u a u^{-1}\right)^{k}=u^{r+2 s}=1\right\} .
$$

In terms of $u$ and $d$ with $d=a u^{-1}$,

$$
G_{1}=\left\{d, u: d^{2}=u^{r+2 s}=\left(u^{-1} d^{-1} u d\right)^{k}=1\right\} .
$$

Next we obtain another quotient group of $G(T(0, n): r, s)$ by adding the relation $t^{k+1}=1$ to (29). After eliminating $t$, we have the group

$$
\begin{aligned}
& \left\{a, u:\left(a^{-1} u_{a u}^{-1}\right)^{k+1}=1, a^{-1} u^{-1} \text { auaua } a^{-1} u^{-1}=1,\right. \\
& \left.u^{r+s}\left[u^{-1} \text { auau }^{-1}\right]^{s}=1\right\} .
\end{aligned}
$$

The second relation is uaua $=$ auau which is the same as $(u a)^{2} u=u(u a)^{2}$. Using this last relation, we may change the third relation to $u^{r-2 s}(u a)^{2 s}=1$. Finally we add the relation $(u a)^{2}=1$ to (31) and obtain the group

$$
G_{2}=\left\{a, u: u^{r-2 s}=(u a)^{2}=\left(a^{-1} u a u^{-1}\right)^{k+1}=1\right\} .
$$

In terms of $u$ and $d$ with $d=u a$,

$$
G_{2}=\left\{d, u: d^{2}=u^{r-2 s}=\left(d^{-1} u d u^{-1}\right)^{k+1}=1\right\} .
$$

Now the groups $G_{1}$ and $G_{2}$ are both instances of the group

$$
\left\{S, T: S^{l}=T^{2}=\left(S^{-1} T^{-1} S T\right)^{p}=1\right\} .
$$

From [3], this group is dihedral if $|l|=2$ and $|p|>1$, is the direct product 
of $A_{4}$ and a cyclic group if $|l|=3$ and $|p|=2$ and is infinite in all other cases with $|l|>1$ and $|p|>1$. Hence (32) is nonabelian unless $|l|=1$ or $|p|=1$. Therefore, since $k$ is positive, $G_{1}$ is nonabelian unless $k=1$ or $|r+2 s|=1$ and $G_{2}$ is nonabelian unless $|r-2 s|=1$. Hence, the groups $G_{1}$ and $G_{2}$ may be used to show $G(T(0, n): r, s)$ is nonabelian except for the case $k=1$ and $|r-2 s|=1$.

Now for the case $k=1$, from (29), $G(T(0, n): r, s)$ is presented by

$$
\left\{a, t, u: t^{2}=u^{-1} \text { tat }^{-1} u t a^{-1}, t=a^{-1} u a u^{-1}, u^{r+s}\left(a u^{-1} t_{t} t^{-1}\right)^{s}=1\right\} \text {. }
$$

Now the first relation is $u^{-1} t a t^{-1}=t^{2} a t^{-1} u^{-1}$. Substituting this into the third relation we obtain $u^{r}\left(a t^{2} a t^{-1}\right)^{s}=1$. (Recall that $u$ and $a t^{2} a t^{-1}$ commute since $u$ is a meridian and $a t^{2} a t^{-1}$ is derived from a longitude.) Now, by adding the relations $t^{3}=\left(a t^{-1}\right)^{2}=1$, we obtain the quotient group

$$
\left\{a, t, u: t^{-1}=u^{-1} \text { tat }^{-1} u t a^{-1}, t=a^{-1} u a u^{-1}, u^{r}=t^{3}=\left(a t^{-1}\right)^{2}=1\right\} \text {. }
$$

Now the first relation is $t^{-1} a t^{-1} u^{-1}=u^{-1} t a t^{-1}$. Substituting $t a^{-1}$ for $a t^{-1}$ twice $\left(t a^{-1}=a t^{-1}\right.$ is a consequence of $\left.\left(a t^{-1}\right)^{2}=1\right)$, the first relation in (33) becomes $t^{-1}=u a^{-1} u^{-1} a$, which is the same as the second relation in (33). Therefore, the first relation in (33) is a consequence of the others. Then, after eliminating $t$, the group (33) becomes

$$
\left\{a, u: u^{r}=\left(a^{-1} u a u^{-1}\right)^{3}=\left(a u a^{-1} u^{-1} a\right)^{2}=1\right\} \text {. }
$$

If we add to this the relation $a^{2}=1$, which implies the third relation in (35), we have the group

$$
G_{3}=\left\{a, u: u^{r}=\left(a^{-1} u a u^{-1}\right)^{3}=a^{2}=1\right\} .
$$

Now $G_{3}$ is nonabelian unless $r= \pm 1$. Hence, the groups $G_{2}$ and $G_{3}$ may be used to show $G(T(0,1): r, s)$ is nonabelian unless $|r|=1$ and $|r-2 s|=1$. Solving simultaneously, we find we are left with just one case, $r=s=1$. Now from (33) and the remarks just after (33), $G(T(0,1): 1,1)$ has the presentation

$$
\left\{a, t, u: t^{2}=u^{-1} t_{t a t}^{-1} u t a^{-1}, t=a^{-1} u a u^{-1}, u=t a^{-1} t^{-2} a^{-1}\right\} \text {. }
$$

Eliminating $u$, we find that the remaining two relations are the same; hence (35) is equivalent to

$$
\left\{a, t: t^{2} a^{-1} t^{-2} a^{-1} t^{2}=a^{-1} t a^{-1}\right\} .
$$

In terms of $t$ and $d$, with $d=a^{-1} t$, this is

$$
\left\{d, t: t^{2} d t^{-3} d t^{2}=d^{2}\right\} \text {. }
$$

Adding the relations $t^{3}=d^{4}=(d t)^{4}=1$ we obtain the quotient 


$$
\left\{d, t: d^{4}=t^{3}=(d t)^{4}=\left(d^{2} t\right)^{2}=1\right\} .
$$

Now from $\S 1.3$ of $[4]$, this is a presentation of the polyhedral group $(4,4 \mid 3,2)$ which is known to be nonabelian. This concludes the proof of Theorem 3.

COROLlary. If $K$ is a knot in $S^{3}$ which has a doubly twisted knot $T(m, n)$ as a companion, with $(m, n) \neq($ even, \pm 1$)$, then $K$ has property $\mathrm{P}$.

Proof. Let $T(m, n)$ be the doubly twisted knot in the solid torus $T$ as shown in Figure 4. $K$ has $T(m, n)$ as a companion means there is an embedding $h$ of $T$ into $S^{3}$ such that $h(T)$ is knotted and $h(T(m, n))=K$. If $K$ does not have property $\mathbf{P}$ then, for some pair $(r, s)$ of relatively prime integers, $M^{3}(K: r, s)$ is simply connected. Hence, by Dehn's lemma, one closed complementary domain of $h(\mathrm{Bd} T)$ is a homotopy solid torus. But one closed complementary domain is $\mathrm{Cl}\left(S^{3}-h(T)\right)$ which is a cube with a knotted hole and the other is $T(T(m, n): r, s)$, neither of which is a homotopy solid torus. Hence $K$ has property $P$.

The class of knots considered in the corollary includes all doubled knots, which were shown, except for zero twists, to have property P by Bing and Martin [2].

\section{REFERENCES}

1. R. H. Bing, Some aspects of the topology of 3-manifolds related to the Poincare conjecture, Lectures on Modern Math., vol. II, Wiley, New York, 1964, pp. 93-128. MR 30 \#2474.

2. R. H. Bing and J. M. Martin, Cubes with knotted holes, Trans. Amer. Math. Soc. 155 (1971), 217-231. MR 43 \#4018a.

3. H. S. M. Coxeter, The groups determined by the relations $S^{l}=T^{m}=\left(S^{-1} T^{-1} S T\right)^{P}$ = 1, Duke Math. J. 2 (1936), 61-73.

4. — The abstract groups $G^{m, n, p}$, Trans. Amer. Math. Soc. 45 (1939), 73-150.

5. H. S. M. Coxeter and W. O. J. Moser, Generators and relations for discrete groups, Springer-Verlag, New York, 1972.

6. J. Hempel, $A$ simply connected 3-manifold is $S^{3}$ if it is the sum of a solid torus and the complement of the torus knot, Proc. Amer. Math. Soc. 15 (1964), 154-158. MR 28 \#599.

7. Jonathan Simon, Methods for proving that certain classes of knots have property P, Ph. D. Thesis, University of Wisconsin, Madison, Wis., 1969.

8. - Some classes of knots with property $\mathrm{P}$, Topology of Manifolds (Proc. Inst., Univ. of Georgia, Athens, Ga., 1969), Markham, Chicago, Ill., 1970, pp. 195-199. MR 43 \#4018b.

DEPARTMENT OF MATHEMATICS, KENT STATE UNIVERSITY, KENT, OHIO 44242 